\title{
Educación Superior en la Unión Europea y el Mercado Común del Sur. Situación hasta 2008
}

\section{Higher Education in the European Union and the Southern Common Market. Situation untill 2008}

Carlos Eduardo Pintos Saraiva*

\section{RESUMEN}

Este trabajo no pretende ser una producción historiográfica de dos Procesos de Integración Regional (PIR), sobre casi medio siglo de acciones en materia de educación dentro de lo que es hoy la Unión Europea (UE) por un lado, y el Mercado Común del Sur (MERCOSUR) por otro; tiene por finalidad, ponderar una aparente falta de medidas en los orígenes de la primera, articular la creación de infraestructuras citando algunas experiencias de ambas, y poder hilvanar la complejidad del todo, para producir algún aporte al estado de la cuestión dentro de la variable educativa en los PIR mencionados. Se centrará el análisis en la Educación Superior de ambas, y rozará el Nivel Medio en Latinoamérica; franja esta, donde la exclusión social replicó especialmente. Cabe aclarar, que cuando se haga referencia al "ahora", "actualmente" 0 "hasta el momento" como límite temporal, estaremos hablando de finales de 2008.

Palabras clave: Unión Europea, MERCOSUR, educación superior

\begin{abstract}
This work is not intended to be a historiographical of two Regional Integration Processes (PIR) on half a century of activities in education within what is now the European Union (EU) on the one hand, and the Common Market South (MERCOSUR ) on the other; the aim is to ponder an apparent lack of action on the origins of the first, joint infrastructure development citing some experiences of both, and to baste the complexity of all, in order to produce a contribution to the state of affairs within the educational variable in PIR mentioned before. Analysis on Higher Education will focus both, and rub the Upper-secondary Level in Latin America; this strip, where social exclusion especially replied. It should be noted that when reference is made to "now", "actually" or "until now" as a time limit, will be talking about the end of 2008 .
\end{abstract}

Key words: European Union, MERCOSUR, higher education

"Universidad Nacional de Tres de Febrero, Universidad Nacional de Avellaneda, Instituto de Enseñanza Integral de Munro, República de Argentina; epsaraiva@untref.edu.ar; epsaraiva@undav.edu.ar 


\section{DESARROLLO}

...si tuviera que comenzar de nuevo, comenzaría por la educación y la cultura...

Jean Monnet

\section{El Contexto Europeo de la Educación Superior}

La universidad europea, que nace en la Edad Media como una comunidad transnacional de estudiantes y profesores, se transforma en el siglo XIX, cuando el nacimiento del Estado-nación da lugar a tres culturas universitarias diferentes, con modelos educativos y estructuras organizativas distintas que actualmente perviven:

1. El modelo alemán: (humboltdiano, centrando el conocimiento y la investigación como objetivos básicos de la universidad) se convierten así en centros de desarrollo científico con escasa (o ninguna) relación con las demandas sociales directas; plasmándose, por ejemplo, en la no existencia de títulos oficiales, cuestión que todavía persiste parcialmente.

2. El modelo francés: (o napoleónico) donde la idea principal es preparar servidores del propio Estado y promover el desarrollo económico de la sociedad, formando a las élites como recurso para ello.

3. El modelo británico: es el que mantiene mejor las tradiciones medievales de formación de los individuos, sosteniendo una menor relación con las necesidades del mercado laboral.

Todos los sistemas europeos de educación superior (también los latinoamericanos) pueden ser clasificados, en alguna medida, dentro de la forma de estos tres modelos con estructuras, planteamientos y objetivos diferentes.

El pasaje de universidad de élite a la sociedad de masas y el arribo a la llamada sociedad del conocimiento, han puesto en evidencia que a dos de los pilares tradicionales de las universidades (investigación y docencia) tuvimos que ańadir el económico. Todo esto, por supuesto, generando profundas transformaciones. 


\section{LA POLÍTICA EDUCATIVA COMUNITARIA ( $Y$ una aparente ausencia de acciones educativas en los orígenes de la UE)}

\section{Creación de "Infraestructuras"}

Para muchos autores, hasta 1970 no se puede hablar de una "política educativa comunitaria", ya que no existía una planificación de directrices que, a mediano o largo plazo, sirvieran de marco o guía para todas las acciones educativas que se pretendían llevar a cabo. Incluso, esa fecha inicial no puede considerarse hasta 1976, año del Primer Programa de Acción en Materia Educativa.

García Suárez, en su obra "Política educativa comunitaria", afirma con rotundidad que: "El indicio precursor de la política educativa comunitaria data del año 1971, cuando una Resolución del Consejo de Ministros de Educación decide crear un centro europeo de educación" (1991: 11).

Resulta interesante también apoyarse en la clásica opinión de Bayona, quien afirma: "El hecho de que la educación, como tal, no aparezca en el Tratado de Roma hizo que desde 1957, fecha de su aprobación, hasta 1971, la educación permaneciera como un tema tabú en la CCE” (1989).

Llega todavía más lejos Rodríguez Carrajo en la interpretación que hace de la frase anterior, afirmando que: "Desde 1951 hasta 1971, la educación constituyó un [tema tabú] dentro del organigrama de la Comunidad Europea. Nadie la mencionaba, nadie pensaba en ella; a nadie se le ocurría hablar de educación como un posible elemento integrador de la Europa comunitaria" (1996: 31).

A partir de lo expuesto, podríamos decir que pareciera no haber existido "política educativa" hasta la aprobación del programa marco de 1976. De hecho, son muchas las explicaciones ofrecidas para justificar esa aparente inhibición, por ejemplo:

a) El carácter exclusivamente económico de los Tratados de París (1951) y Roma (1957) que dan origen a la Unión Europea. 
b) La resistencia de los Estados firmantes de los Tratados a ceder soberanía y, aún más, a cederla en cuestiones tan trascendentes como la educación (agente transmisor de cultura, valores, lengua, ideología), íntimamente ligada a la identidad nacional.

c) Debido a la gran diversidad de y dentro de los países de la UE, la dificultad de materializar procesos de armonización, así como de acciones conjuntas en el terreno de la referencia.

d) La variedad de estructuras político-administrativas en el ámbito educativo que presentan los países miembros.

e) La existencia previa de otros organismos como el Consejo de Europa, quien entre sus competencias tecnológicas tenía la cooperación internacional en materia educativa.

Si analizamos el contexto de la "burbuja educativa", entenderemos por qué funcionaba como un compartimiento estanco. Entre las permanentes pujas de la supranacionalidad y la intergubernamentalidad con la lógica fluctuación; se desarrollaban a principios de los setenta, marcados intentos de fortalecimiento de la unión económica y monetaria. Por otra parte, la crisis del petróleo junto con las relaciones y funcionamiento de la OTAN y la OPEP, jugaron un papel preponderante en cuanto a las prioridades de los miembros.

En la construcción de este andamiaje, y dentro de una idea de inacción, no podríamos dejar de lado el formato lógicamente restrictivo del Tratado de París, centrado en la relación directa materias primas/producción de armamentos. Sin embargo, el siguiente de los Tratados Originarios, el Tratado de la Comunidad Europea de 1957, presenta ya algunos apuntes relacionados a la educación, más precisamente, referidos a la Formación Profesional.

Por tal motivo, sería pertinente efectuar una cita cronológica de las acciones llevadas a cabo, hasta lo que podríamos llamar el "punto máximo" de integración alcanzado en cuestiones educativas, como es la Declaración de Bolonia. A saber:

- Resolución del Consejo de Ministros de Educación, 1971.

- Primer Programa de Acción en Materia de Educación, 1976. 
- Red de Información y Documentación Educativa de la Comunidad Europea (EURYDICE) 1978/79.

- $\quad$ Red NARIC, 1984.

- Programa de Acción, PETRA, 1987.

- Los Programas de Acción EUROTECNETy FORCE, 1989/1990.

- Se crea la "Fundación Europea de Formación" (profesional), 1990.

- Programa Juventud con Europa, 1983/88/90/91.

- Programa COMETT, 1986/88/94.

- Programa ARION, 1987.

- Programa DELTA, 1988.

- Programa LINGUA, 1989.

- Programa ERASMUS, 1989. Extensiones a TEMPUS (países del Este) y ALFA (para Latinoamérica), 1990.

- Tratado de Maastricht,1992 (La Educación como Derecho Primario).

- Libro Verde sobre la Dimensión Europea de la Educación, 1993.

- Programa Leonardo DaVinci, 1994 yLeonardo II (2000/2006).

- El Libro Blanco: Enseñar y Aprender. Hacia la sociedad cognitiva, 1995.

- Programa Sócrates, 1995. Segunda Fase, 2000/06.

- Programa Juventud, 1995.

Las reticencias se podrían explicar desde varios factores. En primer lugar, las diferentes tradiciones educativas de cada uno de los países miembros. Además, la dificultad de armonizar estructuras tan distintas, ya que dichos países representan todos los modelos de sistemas educativos que imperan en Europa: Napoleónico, Anglo-Sajón, Germánico, Centro Europeo y Nórdico. En cuanto a organizaciones administrativas, por ejemplo: los encontramos con diferentes grados de descentralización (Bélgica, Reino Unido y Alemania); centralizadas (Francia); unitarias (Luxemburgo); y con tendencia a la desconcentración (Italia, Holanda, Dinamarca). Consecuentemente, no debemos olvidar que la educación está íntimamente ligada a la política nacional y a la estructura del Estado; los sistemas educativos funcionan como verdaderos órganos de reproducción de pensamiento, de generadores de identidad y formadores de ciudadanos y, la educación, el principal canal de transmisión de la lengua y cultura de un pueblo. 


\section{El Acuerdo de Bolonia}

Tras la eclosión de programas sectoriales que supuso la etapa anterior, se asiste con la firma del Tratado de Maastricht, al inicio de una nueva etapa en la política educativa de UE. A partir de allí, la educación general pasa a ser un Derecho Primario de la Unión.

La inclusión de este artículo supone un replanteamiento global de las acciones en esta materia; debido entre otras cosas, a los numerosos programas existentes hasta ese momento. Cabe destacar, por otra parte, que la inclusión en el tratado de la ciudadanía europea implica un mayor énfasis en políticas que articulan la "Europa de los ciudadanos", por lo que las acciones educativas, sin perder el sentido principal de su relación con el mercado laboral, adquieren una dimensión un tanto más social.

El hecho más trascendente tiene que ver con la educación superior y fue, sin duda, la Declaración de Bolonia, que tuvo lugar en 1999, cuando los ministros de educación europeos (no solo de la EU) se reunieron en esa ciudad y procedieron a su firma.

Esta declaración representó un cambio trascendental en la tradicional política europea sobre educación superior, porque introducía, por primera vez, la idea de "un espacio europeo de educación superior" (EEES).

El intento de materializar la idea del EEES se concreta a partir de la Declaración de La Sorbona (25 de mayo de 1998), coincidiendo con el aniversario de su creación. El proyecto no es propiamente de la UE, pero la arquitectura institucional de esta sirve a los Estados para llevarlo adelante. La iniciativa fue llevada a cabo por los representantes de educación superior de los cuatro países más poblados de la UE: Alemania, Francia, Italia y Reino Unido.

Tan solo un año después, el 19 de junio de 1999, se firma la Declaración de Bolonia, esta vez con el apoyo de 29 países firmantes. La misma hace hincapié en dos cuestiones clave:

1. Recomienda a los países miembros que instauren programas de estudio organizados en tres ciclos:

- Un primer ciclo (tipo Bachelor), cuya duración y características no se especifican, pero debe ser una salida directa al mercado laboral. 
- Un segundo ciclo (tipo Máster), debe ser un ciclo de especialización.

- El tercero, es el tradicional doctorado.

2. Los sistemas de educación superior deberían incrementar:

- Comparabilidad.

- Compartibilidad.

- Transparencia.

- Flexibillidad.

Y se propone una serie de aspectos prácticos, convertidos en seis objetivos esenciales del proceso que se detalla a continuación:

- Adoptar un sistema de títulos fácilmente comprensible y que pueda ser comparable, promoviendo el Suplemento de Diploma.

- Adoptar un sistema estructural para la enseñanza superior organizado en dos ciclos fundamentales.

- Establecer un sistema de créditos compartido (sistema ECTS -European Credit Transfer System-Sistema Europeo de Transferencia de Créditos).

- Promover la movilidad, eliminando los obstáculos para el ejercicio efectivo del libre cambio de estudiantes y profesores, prestando especial atención al acceso a oportunidades de estudio y formación, a los servicios relacionados, al reconocimiento y la valoración de periodos de estudio en el extranjero.

- Promover la cooperación europea para asegurar la calidad educativa en la enseñanza superior.

- Promover la dimensión europea de la enseńanza en el nivel superior.

Todo ello debería estar terminado hacia 2010. Para esto, en el ánimo de seguir impulsando el "Proceso de Bolonia", se fijaron dos nuevas reuniones: una tendría lugar en Praga en 2001, y la otra en Berlín en 2003.

Previo a la reunión de Praga (dos meses antes, 28/29 de marzo de 2001), la mayoría de las instituciones de educación superior de los participantes, se reunieron en la Universidad de Salaman- 
ca, en lo que se llamó Convención de Salamanca y de la cual se extrajeron interesantes aportes.

Así, el 19 de mayo de 2001, en la capital checa, tuvo lugar la primera de las reuniones, sumándose al proyecto, con su firma: Chipre, Turquía y Croacia. En este nuevo comunicado se reafirma la culminación del proceso en 2010, se valoran los avances conseguidos en los seis objetivos, apuntalándolos nuevamente, y se plantean algunas nuevas consideraciones.

La siguiente reunión fue la prevista Conferencia de Ministros de Educación, celebrada en Berlín los días 18 y 19 de septiembre de 2003; ahí se sumaron siete nuevas incorporaciones: Albania, Serbia y Montenegro, Bosnia-Herzegovina, la antigua República Yugoslava de Macedonia, Andorra, Rusia y Vaticano. En la Declaración de Berlín se revisa cada uno de los nueve objetivos marcados hasta entonces y, de todas las propuestas, los Ministros insisten que las prioritarias deben ser tres:

1. Asegurar la calidad.

2. Estructurar el sistema en dos ciclos.

3. Reconocer diplomas y periodos de estudio.

Siguiendo una lógica muy básica de cronología y análisis, arribamos a la Conferencia de Ministros celebrada en Noruega los días 19 y 20 de mayo de 2005: la Declaración de Bergen, donde se incorporan Armenia, Azerbaiyán, Georgia, Moldavia y Ucrania, llegando así a 45 integrantes. Una de las cuestiones más interesantes de ese momento tiene que ver con que, de los 45 integrantes, 36 ya han ratificado la Convención de Lisboa. Queda agendado un nuevo encuentro en Londres para 2007.

A efecto de responder a los retos de un mundo globalizado, el 18 de mayo de 2007 se emite el documento perteneciente a la reunión de Ministros en Londres para verificar los progresos desde Bergen, 2005. En él se da la bienvenida a la República de Montenegro, de lo cual se desprende:

- Los avances se han acercado, en general y significativamente, a la materialización de los EEES.

- Se reitera el compromiso de aumentar la compatibilidad y 
la comparabilidad de los sistemas de educación superior, al tiempo que se recuerda respetar su diversidad.

- Se destacó la importancia de instituciones sólidas, que sean diversas, financiadas adecuadamente, autónomas y responsables.

- Otra de las cuestiones importantes es la transición hacia una educación superior centrada en los estudiantes, y no en una centrada en el profesor.

- Se hizo hincapié en la movilidad de profesores, del PAS, de los estudiantes y titulados, percibiendo como obstáculos cuestiones relacionadas con la inmigración, el reconocimiento de títulos y diplomas, los incentivos económicos insuficientes o las disposiciones rígidas sobre jubilación.

- Asimismo, se reafirmó que "La educación superior debería jugar un papel esencial en la promoción de la cohesión social, en la reducción de las desigualdades y en la elevación del nivel del conocimiento, destrezas y competencias en el seno de la sociedad".

- Dentro de las prioridades para 2009, se debería tener en cuenta:

a) Movilidad.

b) Dimensión social.

c) Recopilación de datos.

d) Empleabilidad.

e) Tratamiento del EEES en un contexto global.

f) Pedido al Grupo de Seguimiento de Bolonia, que continúe con el proceso de balance.

g) Mirar hacia 2010 y más allá.

\section{CONCLUSIONES PRELIMINARES UE}

Ante todo, resultaría pertinente hacer referencia a un pensamiento de José Ortega y Gasset, sobre si existe o no una cultura europea, y sobre el que dio pistas en su obra "Meditación de Europa", allí afirma: "...ninguna nación europea se ha desarrollado ni ha conseguido llegar a su forma plenaria si no es gracias a un fondo ultra o supranacional, que era precisamente la realidad total europea” (1960: 91). 
De todos modos, no resultaría tan fácil declararse un latinoamericano "ultrasupranacionalista", pues ponderando menores diferencias culturales en el espacio latinoamericano que en el europeo, las condiciones actuales difieren tanto en ambas regiones, que la metáfora sería comparable a un púber que está ingresando en la adolescencia con la madurez de los $40 \ldots$

Yendo al punto que nos convoca, podríamos decir:

1. Que luego de este repaso que hemos hecho "a vuelo de pájaro" podríamos afirmar con lo expuesto que, en la UE las políticas en materia de Educación Superior estuvieron presentes desde los primeros tratados fundacionales. Por ejemplo: el propio Tratado EURATOM de 1957, que proponía ya en su artículo No 9 la creación de una institución de nivel universitario que pudiera servir de acicate a la investigación europea del más alto nivel.

2. Que este EEES, es el más ambicioso proyecto de la política educativa de la UE en toda su historia.

3. Que es el resultado del aprendizaje por el intercambio (de profesores y estudiantes).

4. Que surge del entorno (del exterior). No desde dentro de la universidad.

5. Que es un claro ejemplo de un proceso de política educativa.

6. Que esta experiencia transnacional europea de pretendida proyección mundial, posee características diferenciales: porque no se había hecho antes algo similar ni se pretendía en el nivel superior; y por otra parte, tampoco había sido pensado algo parecido para otro nivel educativo.

7. Que, desde una perspectiva comparativa, es interesante destacar que dentro de lo fluctuante del interés de las sociedades por el financiamiento de la educación superior, existía una marcada diferencia de recursos dedicados a ese nivel entre la UE y su gran competidor EEUU, dedicando cifras en torno al $1.1 \%$ y $2.3 \%$ del PBI respectivamente.

8. Que también podemos observar en cuestiones de internacionalización un aspecto interesante, que va de la cooperación a la competencia; puntualmente, entre los actores mencionados ut supra. En este caso, desde 1985 hasta 2000, las universidades europeas descubrieron la importancia de la cooperación 
internacional dentro de la comunidad y también fuera de ella. Pero luego del éxodo de estudiantes europeos a EEUU durante la década de los noventa, se podría percibir a este país como el principal competidor.

9. Que es destacable la gran movilidad de estudiantes dentro de la región, más de un millón aproximadamente (1998-2008) entre los países que la integran. Pero que también es destacable la poca movilidad en el currículum y de los profesores, ya que se puso énfasis en los estudiantes.

10. Que de la relación existente entre la UE y América Latina, la movilidad estudiantil americana es mucho mayor hacia España y Portugal primero, y con la Europa del sur después.

11. Que el Proceso de Bolonia es a la educación, lo que el Euro fue a la moneda respectiva de cada país.

12. Que de todo lo expuesto sobre el Proceso de Bolonia surge, como condición sine qua non, la necesidad de vínculos basados principalmente en un aspecto: la confianza.

\section{El Contexto Latinoamericano/Mercosureño de Educación Superior}

Hasta 1970/1980 (fecha que varía según el país que tomemos), tuvimos en América Latina un escenario universitario predominantemente estatal, con gran autonomía institucional y académica de las universidades públicas. A partir del proceso de reforma universitaria de 1918 iniciado en la provincia de Córdoba, República Argentina, el formato se extendió prácticamente a toda AL.

Un modelo de universidad esencialmente napoleónico, que mostraba una fuerte influencia histórica medieval, con facultades que predominaban (y predominan) hasta por sobre las mismas universidades. Con influencia variable de un país a otro, se suma a esto el modelo de educación superior norteamericana, más precisamente, del modelo americano-canadiense.

Desde la década de los ochenta y básicamente desde los noventa, se producen cambios sustantivos en el escenario político universitario de AL respondiendo al cambio ideológico predominante, donde las políticas neoliberales de "mercado" (desde la versión tan particular y restringida que se dio en la región) reem- 
plazan a las políticas de bienestar. Consecuentemente, se crea un alto número de instituciones universitarias que diversifican fuertemente la educación superior; es decir, se pasó de una educación superior relativamente homogénea a otra con marcada tendencia a la diversificación institucional. Esto devino en una gran heterogeneidad en los niveles de calidad y un gran crecimiento cuantitativo de universidades privadas. En general, es mayor el porcentaje de matrícula en los ámbitos privados que en los estatales. Como rara excepción a este alineamiento, podríamos citar los casos de:

- Argentina

- Uruguay

- México

Un dato interesante para la lectura situacional pueden resultar las siguientes cifras, por ejemplo:

- Pasamos de tener, en 1950, alrededor de 75 universidades en AL, a más de 1500 en la actualidad (dato para el que ni el IESALC -Instituto Internacional de la UNESCO para la Educación Superior en América Latina y el Caribe- puede dar una cifra exacta), debido a la variabilidad del concepto "universidad" que tienen muchos países de la región. Lo que sí podemos afirmar es que se multiplicaron por 20 en los últimos 50 años.

- El número de estudiantes pasó de 260000 en 1950, a aproximadamente 12000000 en 2000; es decir, se multiplicó por 45 en 50 años.

- En los noventa, el crecimiento de la matrícula fue de aproximadamente $8 \%$ (6\% en las universidades privadas y poco más de $2 \%$ en las estatales). Esto explica el crecimiento de la matrícula privada.

Por otra parte, la falta de convergencia en las políticas de educación superior en AL se podría explicar por la influencia de modelos tan distintos, tan puntuales y heterogéneos, sumado a una gran divergencia en términos institucionales y organizacionales. 
En cuanto al diseño de los modelos académicos, se ha producido también un cambio sustantivo: hasta los años 1970/80 existía un predominio de carreras profesionales de modelo napoleónico. A partir de entonces, la ampliación y diversificación de la oferta se genera con muy diferentes criterios académicos. De todos modos, sobreviven las largas carreras tradicionales, las "carreras túneles" en términos de lenguaje europeo, con seis años formales y $8 / 9$ reales. Por otra parte, existen casos donde se acortan las carreras a cuatro ańos, con lo que observamos la supervivencia de dos sistemas.

Asimismo, las carreras se conforman por adiciones, agregados o "parches" que provienen de estos diversos orígenes, con influencias parecidas o hasta diametralmente opuestas. A partir de allí, entonces, encontramos las dificultades, por ejemplo, para compatibilizar y converger, entorpeciendo la movilidad de los estudiantes ante un panorama tan fragmentado.

De ahí la necesidad de debatir sobre objetivos académicos, alcance de los títulos y articulación institucional.

Más recientemente se han desarrollado, con diferente nivel de cantidad y calidad en la región, las carreras de posgrado con gran influencia del modelo de "máster" norteamericano, sin que se haya desarrollado sustancialmente el grado. En varios países, por ejemplo Argentina, las carreras de grado son largas, como así los posgrados, estimándose 8/9 años formales para grado y maestría que se transforman en 12/14 reales siendo, además, muy escaso el nivel de doctorado. Esta situación, por suerte, no es igual en toda la región.

\section{Realidades ipso facto}

- En este contexto de globalización-exclusión es imposible no pensar los procesos sociales en términos globales y regionales, pero con las particularidades latinoamericanas.

- En esta región del planeta, las asimetrías regionales tienen correlato en la educación. Por lo que el proceso está sometido a una marcada heterogeneidad estructural.

- No se puede obviar, bajo ningún punto de vista, la coexistencia de escenarios duales. 


\section{Acuerdos Suscriptos (MERCOSUR Ampliado)}

- Protocolo de Intenciones, 1991.

- Protocolo de Integración Educativa y Reconocimiento de Certificados, 1994; Títulos y Estudios de Nivel Fundamental y Medio no Técnico.

- Protocolo de Integración Educacional, Revalidación de Diplomas, 1995. Certificados, Títulos y de Reconocimiento de Estudios de Nivel Medio Técnico.

- Protocolo de Integración Educacional para el seguimiento de Estudios, 1996. Post-Graduación en las Universidades en los Países del MERCOSUR

- Protocolo de Integración Educacional para la formación de Recursos Humanos, 1996. Nivel de Post-Graduación entre los Países del MERCOSUR.

- Memorando de entendimiento,1998. Sobre la Inserción de un Mecanismo Experimental de Acreditación de Cursos para el Reconocimiento de Títulos de Graduación Universitaria en los Países del MERCOSUR. MEXA.

- Acuerdo de Admisión de Títulos y Grados Universitarios, 1999. Para el Ejercicio de Actividades Académicas en los Países miembros del MERCOSUR, en la República de Bolivia y en la República de Chile

- Acuerdo de Admisión de Títulos y Grados Universitarios, 1999. Para el Ejercicio de Actividades Académicas en los Países Miembros del MERCOSUR.

\section{Plan Operativo del Sector Educativo del MERCOSUR, 2006-2010 (Resumen)}

\section{Características}

- Primera experiencia de integración con un sector ya constituido.

- Posibilitó un espacio diferenciado para la cuestión específica.

- Se constituyó en la idea de generar conciencia social que profundice la democracia y un desarrollo con equidad.

- Idea de papel central en las estrategias de desarrollo. 
EDUCACIÓN SUPERIOR EN LA UNIÓN EUROPEA Y EL MERCADO COMÚN DEL SUR...

\section{Misión}

"Contribuir a los objetivos del MERCOSUR conformando un espacio educativo común, estimulando la formación de la conciencia ciudadana para la integración, movilidad y los intercambios con el objeto de lograr una educación de calidad para todos, con atención especial a los sectores más vulnerables en un proceso de desarrollo con justicia social y respeto a la diversidad cultural de los pueblos de la región” (Plan de Acción, 2001).

\section{Objetivos}

1. Contribuir a la integración regional acordando y ejecutando políticas educativas que promuevan una ciudadanía regional, una cultura de paz y el respeto a la democracia, a los derechos humanos y al medio ambiente.

2. Promover la educación de calidad para todos como factor de inclusión social, de desarrollo humano y productivo.

3. Promover la cooperación solidaria y el intercambio, para el mejoramiento de los sistemas educativos.

4. Impulsar y fortalecer los programas de movilidad de estudiantes, pasantes, docentes, investigadores, gestores, directivos y profesionales.

5. Concertar políticas que articulen la educación con el proceso de integración del MERCOSUR.

\section{Metas (para el nivel superior)}

a) Adopción de un mecanismo de acreditación de carreras en el ámbito del MERCOSUR, tomando en consideración los resultados y lecciones aprendidas del MEXA, y que responda a las exigencias de tiempo y dimensión de cada país.

b) Conocimiento de los sistemas de acreditación de posgrado adoptados por los países.

c) Desarrollo de los vínculos entre universidades y sector productivo. 


\section{Acciones}

a.1) Definir una metodología común de evaluación del MEXA.

a.2) Evaluar el Mecanismo Experimental de Acreditación MERCOSUR (a través de: Seminarios nacionales de evaluación, un Taller regional de evaluación con gobiernos, agencias y actores universitarios) y difundir los resultados de la evaluación con una publicación virtual con la experiencia y las lecciones aprendidas

a.3) Formalizar el Mecanismo de Acreditación del MERCOSUR y aprobar el instrumento jurídico de acreditación de carreras de grado.

\section{Resultados}

- Procedimiento de acreditación de carreras de grado en el MERCOSUR. (Funcionando).

- Sistema de acreditación de estudios de posgrado. (Elaborado).

- Intercambio de experiencias, estudios e investigaciones sobre la calidad de la educación superior. (Realizado).

- Mecanismos de evaluación de la calidad de la educación superior. (En funcionamiento).

- Relevamiento de la oferta y demanda laboral por región. (Efectuado).

\section{Otras Metas (Para el Nivel Superior)}

a) Realización de foros de debate en coordinación con los otros niveles educativos para el intercambio de información, la capacitación y la generación de material didáctico.

\section{Acciones}

a.1) Elaborar los Documentos Base MERCOSUR para ser presentados en los foros. 
a.2) Realizar un foro anual sobre una temática prioritaria para la integración regional (por ej. Historia y Geografía, Derechos Humanos, Medioambiente), convocando a IES y otros actores relevantes.

\section{Resultados}

- Redes de especialistas en Historia y Geografía. (Conformadas).

- Materiales surgidos de Encuentros y Seminarios Regionales de Historia y Geografía. (Difundidos y disponibles en la página web del SIC)

\section{Otras Metas de Superior}

a) Conocimiento de los contenidos académicos, estructurales, culturales y administrativos de cursos de grado existentes en la región para contribuir a hacer efectiva la movilidad y la cooperación solidaria y complementaria.

b) Desarrollo de programas de asociación regional de posgrados para promover la cooperación solidaria y el mejoramiento de la calidad de los mismos.

c) Desarrollo de programas de cooperación interinstitucional que involucren a investigadores, gestores, profesores, estudiantes de las IES.

\section{Acciones}

a.1)Realizar un Foro sobre los desafíos de orden académico, estructural, cultural y administrativo para gestores, académicos, Ministerios de Relaciones Exteriores, de Trabajo y Migraciones.

a.2) Realizar un taller para Meteorología.

a.3)Realizar talleres por área de conocimiento.

a.4)Realizar un Seminario-Taller para el intercambio de información relativa a experiencias vigentes en la región, y para la identificación de áreas comunes de interés.

a.5)Diseñar y constituir un Centro de Estudios e Investigaciones en Educación Superior. 


\section{Resultados}

- Redes de instituciones universitarias de la región. (En funcionamiento).

- Redes de investigadores de la región. (En funcionamiento).

- Centros de cooperación de excelencia entre las universidades de la región. (Funcionando).

- Programas de cooperación entre cursos de posgrados asociados. (Desarrollados).

Ante esta realidad sobrecogedora, que a través de todo el tiempo humano debió de parecer una utopía, los inventores de fábulas que todo lo creemos, nos sentimos con el derecho de creer que todavía no es demasiado tarde para emprender la creación de la utopía contraria.

Una nueva y arrasadora utopía de la vida, donde nadie pueda decidir por otros hasta la forma de morir, donde de veras sea cierto el amor y sea posible la felicidad, $y$ donde las estirpes condenadas a cien años de soledad tengan por fin y para siempre una segunda oportunidad sobre la tierra.

Gabriel García Márquez. La soledad de América Latina. Discurso ante la Academia por la concesión del Premio Nobel.

\section{CONCLUSIONES PARCIALES AL/MERCOSUR}

1. Que existen en la región desde grandes universidades, hasta instituciones tan pequeñas que se las conoce como universidades "garaje". Por supuesto, generando entre otras deficiencias, situaciones diversas en materia de calidad de la educación superior y de su evaluación.

2. Que se debe lograr que los problemas o desafíos de los países, sean desafíos de la región.

3. Que se necesita estudiar y debatir seriamente sobre educación superior, a efecto de facilitar la convergencia y encarar conjuntamente los procesos no regulados de transnacionalización de la educación. Su no regulación adecuada, puede resultar peligrosa. (Referido esto a la gran circulación de personas, bienes y capitales propia de la globalización y las barreras arancelarias o para-arancelarias) 
4. Que ante un escenario tan diversificado y sin olvidar las particularidades de Nuestra América, deberíamos ir pensando en la creación de organismos del tipo ENQA (European Net for Quality Assessment) de Europa, para facilitar los acuerdos regionales sobre el reconocimiento de nuevas instituciones universitarias nacionales y extranjeras.

5. Que la construcción del Espacio Común Latinoamericano de Educación Superior, será muy benéfica si se estudia y se discute en profundidad la experiencia europea y el proceso de Bolonia.

6. Que la existencia de un punto de convergencia a partir de un proyecto regional como es el Proyecto " 6 x 4", es un buen ejemplo de que en AL existe la disposición y preocupación por dar cuenta de los procesos de convergencia desde un espacio propio.

7. Que resulta positivo el proceso inicial que apunta al reconocimiento de los títulos de grado universitarios en los estados miembros del MERCOSUR, más sus dos países asociados: Bolivia y Chile, como el denominado experimento MEXA (Mecanismo Experimental de Acreditación de Carreras), haya dejado de ser experimental y adquiera carácter permanente en 2007.

8. Que coincidimos con lo expuesto por Hans de Wit : “...en América se puede observar que, en este momento, las universidades de la región están cada vez más interesadas en estar vinculadas con el resto del mundo y también entre ellas.”

\section{REFERENCIAS BIBLIOGRÁFICAS}

Boffo, Stefano: "El proceso de Bolonia en Italia". Panel 2 (El proceso de convergencia de la educación superior en Europa), Buenos Aires, EDUNTREF, 2005.

Brunner, José Joaquín. "El Proceso de Bolonia en el horizonte latinoamericano: límites y posibilidades", Universidad Diego Portales, Instituto de Investigación en Ciencias Sociales, Santiago de Chile, 2005.

Coba Arango, Eduardo: "Reconocimiento de títulos". Panel 2 (El proceso de convergencia de la educación superior en Europa), Buenos Aires, EDUNTREF, 2005. 
De Wit, Hans. “América Latina y Europa ante el fenómeno de la internacionalización”, Buenos Aires, EDUNTREF, 2005.

Ferreira Gómes, José. "Nuevas estructuras de estudios". Panel 2 (El proceso de convergencia de la educación superior en Europa), Buenos Aires, EDUNTREF, 2005.

García Suárez, José Antonio. "Política educativa comunitaria" Educación e integración europea, Barcelona, Editorial Boixareu Universitaria, 1991.

Ginés Mora, José y Fernández Lamarra, Norberto (Coordinadores). "Educación Superior, convergencia entre América Latina y Europa" Procesos de evaluación y acreditación de la calidad, Buenos Aires, Comisión Europea/EDUNTREF/ALFA-Europeaid, 2005.

Ginés Mora, José. "La evaluación y acreditación en la Unión Europea”, en Ginés Mora, José y Fernández Lamarra, Norberto (Coords.). Educación Superior, convergencia entre América Latina y Europa. Procesos de evaluación y acreditación de la calidad, Buenos Aires, Comisión Europeal EDUNTREF/ALFA-Europeaid, 2005.

Harris, Nick: "El Proyecto Transeuropa de Evaluación (TEEP)", en Evaluación de la calidad y acreditación. Espacio Común de Educación Superior Unión Europea, América Latina y Caribe (UEALC), Madrid, 2003.

Haug, Guy. "La Declaración de Bolonia y sus consecuencias", II parte, Buenos Aires, EDUNTREF, 2006.

Nieto Nieto, Justo. "El Proceso de Bolonia. Los problemas vistos desde las universidades". Cap 2, Buenos Aires, EDUNTREF, 2005.

Rodríguez Carrajo, Manuel. "Política educativa de la Unión Europea", Salamanca, Universidad Pontificia de Salamanca, 1996.

Valle, Miguel M. "La Unión Europea y su política educativa". Tomos I y II, Madrid, ME y C de España, Centro de Investigación y Documentación Educativa, 2006.

Valle, Miguel M. "Medio Siglo de Acciones en Materia de Educación”. Tomo II, Madrid, ME y C de España, Centro de Investigación y Documentación Educativa, 2006. 